\title{
Exploiting dendritic cells to improve vaccine efficacy
}

\author{
Ralph M. Steinman ${ }^{1}$ and Melissa Pope ${ }^{2}$ \\ ${ }^{1}$ Laboratory of Cellular Physiology and Immunology, Rockefeller University, New York, New York, USA \\ ${ }^{2}$ Center for Biomedical Research, Population Council, New York, New York, USA \\ Address correspondence to: Melissa Pope, Center for Biomedical Research, \\ Population Council, 1230 York Avenue, New York, New York 10021, USA. \\ Phone: (212) 327-7794; Fax: (212) 327-7764; E-mail: mpope@popcbr.rockefeller.edu. \\ J. Clin. Invest. 109:1519-1526 (2002). doi:10.1172/JCI200215962.
}

The challenges to vaccine biology are dramatized by the current situation with an AIDS vaccine (see Letvin, this Perspective series, ref. 1). For years, data have been available on the HIV-1 genome and its proteins, as well as numerous antigens recognized by the immune system. Still, this information has not been readily translated into candidate vaccines that induce the broad and longlasting $\mathrm{T}$ cell-mediated immunity thought to be necessary to protect people from acquiring AIDS (2-5). Vaccines are also lacking for many other serious infections in which $\mathrm{T}$ cell-mediated immunity should be protective. These include pathogens whose genomic sequences and antigenic proteins are well characterized: tuberculosis, malaria, and the herpes simplex, papilloma, Epstein-Barr, and hepatitis C viruses. In essence, the identification of foreign antigens is necessary but not sufficient for producing vaccines that are effective in the $\mathrm{T}$ cell sphere. Better vaccine delivery and vaccine adjuvants, or enhancers of immunity, are required $(6,7)$.

We propose that dendritic cell (DC) physiology should be considered and exploited in meeting each of the challenges in vaccine biology that lie ahead (see Table 1). DCs act as nature's adjuvants for regulating antigen-specific immunity. As antigen-presenting cells, DCs capture antigens, process them into peptides, and present them on products of the MHC to T cells. DCs are both efficient and specialized in antigen presentation, and they control the magnitude, quality, and memory of the ensuing immune response. DCs have been used successfully as cellular adjuvants in mice to elicit protective $\mathrm{T}$ cell-mediated immunity against pathogens and tumors $(8,9)$. These cells are now being used to prime and expand $T$ cells specific for human cancers (refs. 10-12; see also Yu and Restifo, this Perspective series, ref. 13). The responding $T$ cells include helper cells, especially $\mathrm{Th} 1 \mathrm{CD} 4^{+}$cells, which produce IFN- $\gamma$; and killer cells, especially $\mathrm{CD}^{+}$cytolytic T lymphocytes (CTLs), which exocytose granules rich in perforin and granzyme. New information indicates that DCs control responses by other classes of lymphocytes (B, NK, and NKT cells) and elicit T cell memory, a critical goal of vaccination.

Developing the capacity to harness DCs for vaccination seems particularly urgent in confronting infectious agents that, like HIV-1, pose unusual demands with respect to safety; the time-honored approach of microbial attenuation is now being set aside as vaccine biologists turn to defined antigens, poorly replicating vectors, and DNA. Although these vaccines introduce foreign microbial products, they often generate weak immunity, especially $\mathrm{T}$ cell-mediated immunity. Consequently, greater emphasis on underlying immunologic processes is needed, notably the strong adjuvant roles of DCs. Interestingly, as we discuss below, even the classical vaccine approach of microbial attenuation, used successfully for smallpox and measles, may have unknowingly exploited the adjuvant roles of DCs.

\section{DCs as natural adjuvants}

In vitro studies. DCs are potent stimulators of $\mathrm{T}$ cell responses and T-dependent antibody formation in tissue culture. Relatively few DCs and relatively low doses of antigen are required to elicit high levels of lymphocyte proliferation and differentiation. Initially, because DCs had to be isolated directly from lymphoid tissues (or, in the case of humans, from blood), the scarcity of these cells imposed a serious limitation on DC research. Typically, DCs make up less than $1 \%$ of a given cell population - a figure that is somewhat misleading since the frequency of these cells is at least 100 times greater than that of $\mathrm{T}$ cells specific for any given antigen. Moreover, DCs are extensively ramified in regions of the lymph nodes through which $T$ cells recirculate (Figure 1).

Most investigators now study DCs produced in much larger numbers from either $\mathrm{CD} 34^{+}$proliferating progenitors or $\mathrm{CD} 14^{+}$nonproliferating monocytic precursors. These DCs are charged or "pulsed" with antigens, which they efficiently process and display as MHC-peptide complexes. Antigen-pulsed DCs can be placed into culture with lymphocytes, whereupon $\mathrm{T}$ cells begin to proliferate and to produce lymphokines and various cytotoxic molecules. Primary responses to microbial antigens can be difficult to achieve in short-term (1 week) culture, because the initial number of antigenresponsive cells is so low ( $<1$ in $10^{5}$ lymphocytes), but mature DCs rapidly induce recall responses to many antigens, including those from HIV-1 and influenza. These viral antigens are presented to primed $\mathrm{CD}^{+}$and 
$\mathrm{CD}^{+} \mathrm{T}$ cells even when delivered to the DCs in poorly replicating vectors and as ultraviolet light and chemically inactivated virions. The potency of DCs in stimulating $\mathrm{T}$ cells in vitro reflects both their specialized ability to capture and present antigens and the effects of other molecules, not present in MHC complexes, that enhance $\mathrm{T}$ cell binding and stimulation.

In vivo evidence for $D C$ as strong adjuvants. Vaccination with DCs leads to protective immunity against infections and tumors $(8,9)$ and, in the case of certain self antigens, autoimmunity. DCs can be exposed to an antigen either in vivo, by introducing the antigen directly, or ex vivo, by pulsing the cells with antigen while they are in culture and administering them to genetically matched animals. After antigenic proteins are given to mice, DCs are found to be the main cells capturing antigen in an immunogenic form. When mice are challenged with microbes, DCs also are the principal cells producing the key protective cytokine IL-12. Ex vivo-activated DCs can prime recipient animals in an antigen-specific manner, allowing them to respond to an antigenic challenge within a week. These DCs migrate to the recipients' lymph nodes and lodge in the $\mathrm{T}$ cell areas, sites through which lymphocytes enter the lymph nodes via high endothelial venules (Figure 1). This movement positions the DCs in a seemingly ideal niche to select antigen-specific $T$ cells when the latter percolate through the node. Such selection can be observed directly in situ: Following activation in contact with DCs, the T cells leave the lymph node, freeing them to fight infections and tumors. Some also become memory $\mathrm{T}$ cells, a response whose mechanism remains to be unraveled.

For purposes of vaccine design, it may be most straightforward to target antigens selectively to DCs in situ. This has been achieved through the DEC-205 receptor (CD205) (14), which mediates antigen uptake and processing in DCs. Crucially, induction of immunity also requires a stimulus that matures the DEC $-205^{+}$DCs. Antigen presentation sets the stage for antigen-specific $T$ cell recognition, but maturation controls the $\mathrm{T}$ cell response. Therefore, vaccines must not only contain the requisite antigens to initiate protective immunity but also provide stimuli to promote DC maturation.

\section{Exploiting the adjuvant roles of DCs}

To date, the role of DCs in vaccine efficacy has been studied in detail only in mice receiving DNA vaccinations. Nevertheless, it is already evident that DCs have several features that could be modulated using appropriately designed vaccines to generate stronger $T$ cell-mediated immunity. Below, we consider three aspects of DC biology that are of particular interest in vaccine development: antigen presentation, DC maturation, and DC mobilization.

Antigen handling and presentation. Vaccine antigens are not presented directly to the immune system but must first be captured, processed, and bound to antigen-presenting molecules, typically those of the class I or class II MHC. Humoral immunity depends on the fact that
B cells and their antibody products react directly with native antigens on pathogens or their toxins, thus neutralizing the pathogen or toxin extracellularly, prior to binding and/or entry into cells. In contrast, $T$ cell-mediated immunity to intracellular infections requires recognition of fragmented antigens produced within infected targets. The fragments are typically peptides that bind to highly polymorphic class I and class II products of the MHC and are then displayed on the cell surface as MHC-peptide complexes (15). Other, less polymorphic antigen-presenting molecules have been found, including the CD1 family, which is responsible for the presentation of microbial glycolipids (16), and the so-called MHC class Ib products, which present formylated bacterial peptides (15).

Despite the fact that DCs can capture and present to $\mathrm{T}$ cells even nonspecific, soluble proteins that prove to be poorly immunogenic, DC targeting clearly offers a valuable strategy for vaccination. Quantitative efficiency is one significant benefit of this approach $-a$ peptide sequence within a protein delivered specifically to DCs is $100-1,000$ times more efficient than a peptide given in a nonspecific adjuvant like CFA (14). A second benefit relates to the quality of the antigen processing (17). For example, by targeting select antigen uptake receptors on DCs, the vaccine can access their more efficient antigen processing and presentation pathways, particularly the exogenous pathway discussed below, which allows proteins and poorly replicating vaccines to load both MHC class I and class II molecules, as well as CD1.

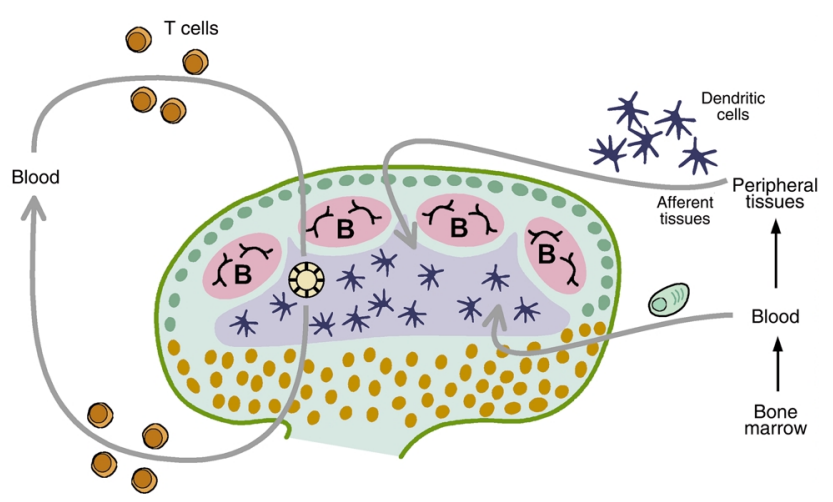

\section{Figure 1}

Lymphocyte and DC circulations. Naive lymphocytes circulate from blood via high endothelial venules into lymphoid tissues. B cells then move into follicles while T cells percolate through $T$ cell areas, both eventually leaving the node via efferent lymphatics to return to the blood. Upon antigen recognition, some activated $B$ and $T$ cells, as well as DCs and follicular dendritic cells (a distinct cell type that, unlike DCs, retains native antigens as immune complexes), congregate in the follicles to generate the germinal center reaction for antibody formation. Other activated $B$ and $T$ cells return to inflammatory sites via the blood or become memory cells. Some of the latter are termed "effector memory" cells, because they can rapidly produce cytokines and are positioned in peripheral tissues. In parallel to the circulation of lymphocytes, DCs move from blood to tissues and then into afferent lymphatics, which bring DCs into the T cell areas where they eventually die. The plasmacytoid subset of DCs enters the $T$ cell areas directly from blood; their subsequent fate is unclear. 
Table 1

Challenges in vaccine biology requiring improved control of antigen presentation

Enhance antibody and $T$ cell-mediated immune memory, especially in humans

Improve the quality of the $T$ cell response, e.g., CD4 ${ }^{+}$Th1 helper and CD8 ${ }^{+}$killer cells

Achieve mucosal immunity, particularly for sexually transmitted diseases

Design therapeutic as well as preventive vaccines, e.g., against HIV-1 and cancer

Identify vaccines that dampen immunity, e.g., in autoimmune disease

Antigen presentation on MHC class I products, including the exogenous pathway. The presentation of vaccine antigens on MHC class I is needed to activate $\mathrm{CD}^{+} \mathrm{CTLs}$, which kill infected targets early in the microbial life cycle, thereby blocking replication and spread of the pathogen. The classical, "endogenous" pathway for presenting peptides on MHC class I products begins when DCs or other cells are productively infected, as when DCs present antigens from influenza and recombinant vaccinia virus. Following endogenous synthesis within DCs, microbial proteins are clipped by the proteasome, and peptide fragments are transported via transporters for antigen presentation (TAPs) into the rough endoplasmic reticulum (15). There, the resulting peptides are affixed to the peptide-binding grooves of newly formed MHC class I products, and the MHC-peptide complexes exit via the Golgi apparatus to reach the surface for presentation to antigen receptors on T cells (15).

DCs are proving to be quite specialized in their capacities to form MHC class I-peptide complexes, which go beyond the classical endogenous pathway summarized above. One specialty is to present viruses that have been inactivated by ultraviolet light, heat, or chemical treatment, responses not seen with most other cell types. The inactive viruses retain their capacity to fuse with the plasma or endocytic vacuole membrane, thereby delivering some virion proteins into the cytoplasm. Subsequent efficient processing of incoming virions, or processing of newly synthesized proteins produced at low levels, may explain the capacity of DCs to present inactivated but fusogenic viruses, but this possibility needs further study.

Another specialty of DCs is to bring about what is termed "exogenous presentation" or "cross-presentation." These pathways act, respectively, on proteins derived from immune complexes or inactivated microbes, or on antigens originally synthesized in other cells, which then "cross" to the MHC products of DCs. In all such cases, antigens depend on selective endocytic uptake receptors to gain access to the cytoplasm of DCs, after which they likely engage the known DC systems that allow for protein ubiquitination, proteasomal cleavage of antigens, and TAP-mediated peptide transport. Thus, endocytosed antigens can gain access to the cytoplasm without the need for a viral envelope to mediate delivery. The exogenous pathway allows DCs to present many forms of nonreplicating antigens on MHC class I and thereby to elicit $\mathrm{CD}^{+} \mathrm{CTLs}$. Active infection and biosynthesis do not need to take place in the DCs $(18,19)$. A good example is vaccinia virus: This prototype for successful vaccines is actually presented, at least in mice, almost entirely by the exogenous or cross-presenting routes (20).

Several DC receptors lead to MHC class I-peptide complex formation via the exogenous pathway. These include the Fc $\gamma \mathrm{R}$, which binds immune complexes and antibody-coated tumor cells; the integrin $\alpha_{v} \beta_{5}$ and the phosphatidylserine receptor, which bind dying cells; and various receptors for heat shock proteins. Subsequent delivery of antigen into the cytosol is postulated to require a transporter that allows macromolecules to escape the endocytic vacuole. Once in the cytoplasm, proteins may be subject to the newly recognized heightened capacity of maturing DCs to polyubiquitinylate proteins. Ubiquitin conjugation marks the proteins for efficient proteasomal processing. It is anticipated that additional DC specializations will be found for increasing their efficiency in MHC I-peptide complex formation.

The exogenous and cross-presentation pathways via DCs constitute important routes to natural immunity in many infectious diseases, because DCs can capture and present antigens from immune complexes or dying infected cells to elicit $\mathrm{CD}^{+} \mathrm{T}$ cell immunity $(18,19)$. These pathways also substantially change how one approaches the design of vaccines for cell-mediated immunity. Nonreplicating vaccines, such as protein subunits and chemically inactivated vaccines, are generally thought to be unable to elicit CD8 ${ }^{+}$CTLs, which may be critical for defense against certain chronic intracellular infections and tumors. Subunit and inactive vaccines lose efficacy, it is thought, because they do not lead to the new intracellular synthesis of proteins required for processing in the classical endogenous pathway to MHC class I. However, the barrier to developing vaccines that engage the class I MHC seems no longer insurmountable if immunologists can learn to exploit the exogenous pathway in DCs.

It should be noted that, although many investigators use the terms "exogenous pathway" and "cross-presentation" to refer exclusively to presentation on MHC class I, DCs simultaneously present exogenous proteins and cellular antigens on MHC class I and II. Thus, as considered below, $\mathrm{CD}^{+}$helper $\mathrm{T}$ cells can be engaged to amplify the $\mathrm{CD}^{+} \mathrm{T}$ cell-mediated, MHC class I-restricted responses initiated by DC presentation.

Antigen presentation on MHC class II products. The MHC class II pathway, which forms MHC-peptide complexes to be recognized by $\mathrm{CD}^{+}$helper T cells, is particularly efficient in DCs. To illustrate, when a protein is delivered to DCs from dead cells, the formation of MHC II-peptide complexes is actually many thousand times more 
efficient than when preprocessed peptides are applied. DCs have many candidate receptors for dying cells (21), but active receptors in vivo remain to be identified. Conversely, several DC-restricted uptake receptors are known (Figure 2) for which natural ligands remain to be identified. One example is the DEC-205 (CD205) uptake receptor, which traffics in a distinct way through DCs and greatly enhances antigen presentation relative to other adsorptive endocytic receptors. DEC-205 can recycle through the acidic late endosomal/lysosomal vacuoles in maturing DCs, compartments that are enriched for MHC class II molecules and proteinases like the cathepsins that mediate antigen processing and MHC class II-peptide complex formation.

These complexes, once formed, are transported to the DC surface within distinctive nonlysosomal transport vesicles. The vesicles contain both the MHC-peptide complexes, recognized by the $\mathrm{T}$ cell receptor, and the CD86 molecules, required to costimulate $T$ cell growth. Upon arrival at the DC surface, the processed antigen and CD86 remain coclustered in aggregates that contain so-called tetraspannin membrane proteins. This situation seems ideal to set up immunologic synapses between DCs and the T cells that they activate. At this final mature stage, the DCs silence transcription of MHC class II products (whose genes are activated by the transcriptional activator CIITA) and shut down much of their endocytic activity, while actively presenting antigens captured in the periphery or vaccine site at lymphoid tissues (Figure 1).

\section{Antigen presentation on CD1 glycolipid- binding molecules}

DCs express the known members of the CD1 family of antigen-presenting molecules, but individual CD1 molecules can be restricted to subsets of DCs. CD1a is typically found on epidermal Langerhans cells in skin, while CD1b and c are expressed on dermal DCs. CD1 molecules present microbial glycolipids (22), but in addition, CD1d on monocyte-derived DCs presents the drug $\alpha$-galactosylceramide. The CD1d-restricted cells are called NKT cells. Following recognition of glycosphingolipid on CD1d, NKT cells orchestrate the production of large amounts of cytokines from several cell types and have the capacity to act as adjuvants for $\mathrm{T}$ cell-mediated immunity (23). Interestingly, none of the CD1 molecules have been found on the plasmacytoid subset of DCs discussed below.

$D C$ maturation. In the absence of a perturbation such as infection or vaccination, most DCs remain at an immature stage of differentiation. To exploit DCs in vaccine design, the vaccine must not only provide protective antigens that are captured by DCs; it must also induce DC maturation.

Immature DCs can capture antigens, but they must differentiate or mature to become strong inducers of immunity. DC maturation is the control point that determines whether an antigen is to become an immunogen, and it can take place not just as a response to microbial infections, but also in other forms of strong $\mathrm{T}$ cell-mediated immunity such as transplantation reactions, contact allergy, and autoimmunity. There are two well-studied classes of maturation stimuli. One class is provided when the microbe or vaccine signals DCs through toll-like receptors (TLRs); a second class is provided by lymphocytes and other cells (either T, B, NK, NKT, platelets, or mast cells) that deliver TNF-type signals to the DCs.

Many defined microbial products initiate DC maturation through TLR signaling $(24,25)$. Cytokine production is triggered quickly, as is also the case with many other cell types. However, DCs can produce particularly high levels of immune-enhancing cytokines like IL-12, IFN- $\alpha$, and even, in some situations, IL-2. Over longer periods, DCs mature to become strong adjuvants for $\mathrm{T}$ cell immunity. Expression of specific TLRs can be high in DCs, particularly TLR9, which responds to microbial DNA (26), and TLR3, a receptor for double-stranded RNA. TLRs can respond to particular small molecules, like specific $\mathrm{CPG}$ deoxyoligonucleotide sequences, or to complex microbial macromolecules like DNA. As discussed below, distinct DC subsets express different complements of TLRs. In terms of signal transduction, TLRs use the MyD88 adaptor protein to trigger cytokine release from different cell types (24). However, DC maturation through certain TLRs is also influenced by a MyD88-independent mechanism $(24,25)$ that will be important to identify and manipulate.

TNF family members that stimulate DCs include TNF itself, Fas ligand (FasL), CD40 ligand (CD40L), and TRANCE (RANKL). These molecules are expressed in a membrane-bound form by activated $\mathrm{T}$ cells and signal the corresponding activating subclass
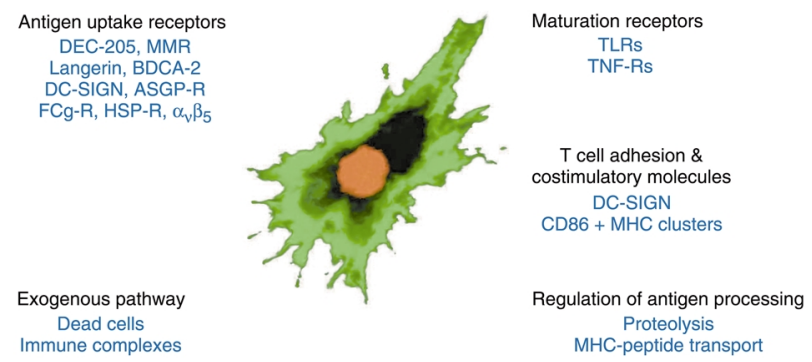

\section{Figure 2}

Some specializations of DCs for vaccine capture, MHC-peptide complex formation, and T cell stimulation. DCs express many adsorptive uptake receptors whose natural ligands are generally not yet known. For this reason, anti-receptor antibodies are often used experimentally as surrogate antigens. Several receptors are type II transmembrane proteins with a single external C-type lectin domain found on distinct DC subsets: DC-SIGN and the asialoglycoprotein receptor on monocyte-derived DCs, BDCA-2 on plasmacytoid cells, and Langerin on Langerhans cells. MMR and DEC-205 are type I proteins with eight to ten contiguous C-type lectin domains; these receptors can also be expressed on certain endothelia and epithelia. Other receptors, such as Fc $\gamma \mathrm{R}$, are not DC-restricted but function selectively in DCs to mediate the exogenous pathway for presentation on MHC class I products. Beyond antigen capture, DCs (or particular DC subsets) express high levels of select TLRs and thereby mature in response to specific microbial stimuli. During maturation, DCs produce and export high levels of several costimulatory molecules for $T$ cell growth and differentiation. DC maturation regulates many of the elements involved in antigen capture and processing. 
of TNF receptors (TNF-Rs). When microbial or vaccine stimuli mature DCs, CD40 and TRANCE receptor (TRANCE-R) are induced. As a result, once antigencapturing DCs reach the lymph node (Figure 1), control of DC function can switch from the microbe to the T cell. Possibly, different maturation stimuli (TLR signaling via the microbe, for instance, rather than TNF-R signaling via the T cell) have different consequences for DCs. Full expression of some DC functions, such as IL-12 production, may also require concerted signaling by both of these receptor types.

DC maturation is an intricate differentiation process whose different components may be under separate control. Antigen processing and presentation are regulated at several levels, notably through the control of intracellular proteinase activity. Thus, maturation diminishes the level of the cysteine protease inhibitor cystatin $\mathrm{C}$ within the endocytic system, permitting increased catabolism of the invariant chain by cathep$\sin \mathrm{S}$, and promoting the binding of antigenic peptides to MHC class II molecules. The expression of CD40 and other $\mathrm{T}$ cell interaction molecules is also enhanced by maturation. Signaling through CD 40 , induced by CD40L on activated $\mathrm{T}$ cells, mast cells, and platelets, leads to the production of DC cytokines and chemokines and enhances DC migration and survival. Maturing DCs alter their expression of the costimulatory molecules CD80 and CD86 and of TNF family members, all of which can influence the extent and quality of the immune response. Maturing DCs also reshape their repertoire of chemokine receptors (27). Mature DCs lose CCR5 and CCR2, which respond to chemokines in an inflammatory site, but gain CCR7, which responds to chemokines in the lymphatic vessels and lymphoid organs. Maturation nicely illustrates the importance of taking a DC perspective in vaccine design. By targeting a vaccine to immature DCs and also maturing the cells, one implements a large spectrum of features (from antigen handling to proper positioning in vivo to optimal control of the magnitude and quality of the immune response) conducive to strong antigen-specific immunity.

Many existing vaccines may induce DC maturation, although their mechanisms can be quite complex. Some current vaccine vectors - recombinant yeast vaccines and DNA vaccines (28) among them - induce DCs to become strong stimulators of immunity, probably by directly stimulating TLRs. The attenuated smallpox and measles vaccines appear to mature DCs in a different manner. These organisms are both infectious and cytotoxic for DCs and yield infected dead cells that can then be processed efficiently through the exogenous pathway in other DCs. Furthermore, perhaps through the release of heat shock proteins, dying cells can mature the antigen-capturing DCs. Thus, vaccines may produce stronger immunity when they initially kill some DCs.

Effects of DC mobilization. DC mobilization entails both an increase in the population of these cells and a change in their migratory properties $(27,29)$. DC numbers can be increased tenfold using cytokines like
G-CSF and flt-3L, while DC differentiation from nonproliferating precursors can be influenced by other hematopoietins (GM-CSF, IL-4) and IFNs. The requisite chemokine receptors for DCs to traffic into a vaccination site may vary, with CCR6 likely responding to macrophage inflammatory protein $3 \alpha$ (MIP-3 $\alpha$ ) at mucosal surfaces, and CCR5 and CCR2 responding to MIP-1s and monocyte chemoattractant proteins in other interstitial compartments. For vaccines administered into the skin and muscles, migration to lymph nodes requires afferent lymphatics $(30,31)$, but the DC-lymphatic interaction is still poorly understood. DCs must also migrate in a directed way to the $T$ cell areas, responses that are influenced by cysteinyl leukotrienes and transporters of the multidrug resistance family, as well as the distinct TREM-2 signaling molecule, each of which acts on the CCR7 lymph node homing receptor on DCs. Once in the T cell area, DCs are short-lived, apparently dying within a few days. Their lifespans can be prolonged through membrane bound TNFs on the T cell, e.g., CD40L and TRANCE (RANKL).

In summary, we propose that vaccine efficacy or immunogenicity can be improved by altering DC functions at three levels: by enhancing vaccine capture and processing, by promoting DC maturation, and by increasing DC numbers by stimulating DC replication, survival, and migration, to the lymph nodes.

\section{Other endpoints in the immune response that can be achieved via DCs}

Improved cell-mediated responses and T cell memory. Antigen-primed DCs rapidly prime an individual to form IFN- $\gamma$-producing or Th1CD4+ helper cells. When DC maturation is blocked, IL-4- and IL-5-producing Th2 helper cells seem to be induced, resulting in less efficient $\mathrm{T}$ cell-mediated immunity and memory, as well as the production of undesirable antibody subclasses - notably IgEs, which mediate allergy. Th1 helpers are especially critical in activating macrophages to resist intracellular bacteria and protozoa, and they are also the most effective form of helper for $\mathrm{CD}^{+} \mathrm{CTL}$ resistance to experimental viral infections and tumors. $\mathrm{CD}^{+}$Th1 cells additionally render DCs resistant to killing by $\mathrm{CD}^{+}$CTLs and directly lyse important MHC class II-expressing infected cells through a FasLdependent (rather than perforin/granzyme-dependent) mechanism. The induction of Th1 cells is often ascribed to IL-12, but mature DCs can lose high-level IL-12 production while maintaining their ability to induce strong $\mathrm{CD} 4^{+} \mathrm{Th} 1$ and $\mathrm{CD} 8^{+} \mathrm{CTL}$ responses in vivo, possibly through other cytokines or special B7 and TNF family members.

When DCs directly stimulate CD8 ${ }^{+}$CTLs in humans, the $T$ cells can kill targets in the presence of lower doses of peptide; in this way, the functional affinity of the $\mathrm{CD}^{+} \mathrm{T}$ cell is improved. A recent intriguing mechanism for this is that antigen-reactive $\mathrm{T}$ cells somehow remove MHC-peptide from the DCs, favoring selection of the more competitive, higher-affinity $\mathrm{T}$ cells. Importantly, DCs induce $\mathrm{T}$ cell memory for both high-affinity $\mathrm{CD}^{+}$and $\mathrm{Th} 1 \mathrm{CD} 4^{+}$responses. 
Generation of antibody-forming B cells. Classically, DCs enhance antibody formation by promoting the formation of antigen-specific $\mathrm{CD}^{+}$helper $\mathrm{T}$ cells, which induce antigen-specific $\mathrm{B}$ cells to proliferate and make antibody. In situ, IFN- $\alpha$ enhances T-dependent antibody formation, isotype switching, and memory. To obtain this result, DCs are the only cells that need to express the requisite type I IFN receptors. DCs can have direct effects on B cells that greatly enhance Ig secretion and isotype switching, including the production of the IgA subclass of antibodies, which contribute to mucosal immunity. Recently, DCs have been pulsed ex vivo with cell wall constituents from Streptococcus pneumoniae. When the DCs are reinfused into genetically matched mice, strong T-dependent B cell responses are induced to microbial proteins and capsular polysaccharides. This requires IL- 6 production by the DCs and quite possibly a ménage à trois wherein $\mathrm{B}$ and $\mathrm{T}$ cells respond to native and processed antigens being presented on the same DC or DC subset. Consequently, if vaccines are delivered to the appropriate DCs, combined $\mathrm{B}$ cell and $\mathrm{T}$ cell immunity can ensue, an important consideration in the context of HIV-1 and other chronic infections.

Implementation of mucosal immunity. Vaccines are lacking for many sexually transmitted diseases. Strong $\mathrm{T}$ cell immunity and IgA antibodies may be required to provide protection from HIV-1, Epstein-Barr, herpes simplex, and human papilloma viruses. DCs are located beneath the antigen-transporting epithelium (M cells) (32) of mucosal lymphoid organs, and they may extend their processes through standard, lining epithelia to capture antigens. Access of vaccines to mucosal DCs should prove valuable for inducing mucosal immunity. However, maturation is again likely to be needed. Some mucosal DCs in the steady state produce IL-10 and may induce regulatory or immunosuppressive $\mathrm{T}$ cells, as discussed below. The latter would compromise vaccine efficacy.

\section{Newly appreciated features of DCs relevant to vaccination}

$D C$-induced tolerance. The immune-enhancing or adjuvant roles of DCs are exerted in two phases. In the first immediate or innate phase, DCs capture the antigen, begin to mature in response to stimuli, particularly microbial components, and produce cytokines and chemokines that mobilize and differentiate other cells, including NK cells. In the slower (adaptive) phase, DCs stimulate several components of the $\mathrm{T}$ cell response: clonal expansion; differentiation, specifically into Th1 helper or killer cells; and memory. Much of current effort by DC biologists focuses on a different effect of these cells, namely their capacity, when in the immature state, to induce antigen-specific unresponsiveness or tolerance following antigen capture.

DCs in the steady state are immature and can silence immunity in an antigen-specific manner through two recently identified mechanisms (33). Here, the "steady state" refers to the absence of acute inflammation and infection, with the latter providing stimuli that mature
DCs via TLRs and TNF-Rs. One tolerance mechanism is exerted by DCs bearing the DEC-205 receptor in the lymph nodes. When antigen is targeted to these DCs in the absence of a maturation stimulus, interacting $\mathrm{T}$ cells proliferate but are soon deleted. Effector functions (IFN- $\gamma$ secretion) and memory therefore do not develop, and the animal becomes tolerant to rechallenge with the peptide in a strong adjuvant (14). In contrast, chronic stimulation of DCs may predispose to the development of autoimmunity, including lupus erythematosus. A second tolerance mechanism involves the induction by certain immature DCs of IL-10-producing $T$ cells, which in turn can silence other effector $\mathrm{T}$ cells. The tolerogenic roles of DCs could compromise vaccine efficacy. Conversely, the capacity to induce regulatory $\mathrm{T}$ cells may be useful in the design of a new class of vaccines for suppressing immunity in autoimmune diseases, allergy, and transplantation.

Contributions of DC subsets to innate immunity. There are many different forms of DCs in situ. These comprise the Langerhans cells in the skin and other epithelia and various DC precursors in blood, including monocytes and plasmacytoid DCs. Many DCs move from blood to tissues to lymph and then to the lymph node, but plasmacytoid DCs can move from blood directly into the lymph node via high endothelial venules, presumably by virtue of their CD62L expression (Figure 1). A hallmark of the plasmacytoid DC is the capacity to produce prodigious levels of IFN- $\alpha$ upon challenge with many viruses.

The raison d'être for these DC subsets has been a mystery, but some recent findings provide new perspectives. DC subsets vary in their expression of TLRs and therefore respond to different microbial stimuli. For example, CD11 $\mathrm{c}^{+} \mathrm{CD} 14^{-}$cells in blood are the principal expressers of TLR3, a receptor for double-stranded viral RNA, whereas plasmacytoid cells are the main cell in blood expressing TLR9, the receptor for bacterial DNA and specific CPG deoxyoligonucleotides (26). Furthermore, distinct DC subsets can produce large amounts of individual cytokines (IL-12, TNF, IFN- $\alpha$ ) in response to TLR signaling. This, in turn, may influence the kinds of lymphocytes (Th1 helpers, $\mathrm{CD}^{+} \mathrm{CTLs}$ ) that are expanded by the antigen-presenting DCs. Importantly, because DC subsets also express different endocytic receptors, it may be possible to design selective vaccines targeting Langerhans cells, plasmacytoid DCs, or monocyte-derived DCs (Figure 2). As immune responses to specific pathogen-associated antigens are elucidated, it may prove important to target vaccines through the appropriate DC subset to take advantage of their distinct pathways for antigen uptake, maturation, and cytokine release.

\section{DCs in the response to DNA vaccines}

DNA vaccines are now showing promise in priming for resistance to simian immunodeficiency virus. At face value, the efficacy of DNA vaccines is perplexing, since the vaccine proteins are expressed primarily within skin or muscle cells, which are weak antigen-presenting cells 
for inducing immunity. However, successful DNA vaccination may involve DCs (28). A few DCs may be directly transfected with the vaccine and be responsible for immune priming. DCs may also capture antigens expressed in other cells that die following transfection and protein expression. Importantly, DNA itself activates many other DCs that are not transfected, most likely via TLR9 (26). Unfortunately, the requisite DNA receptors (and their CpG deoxyoligonucleotide mimics) may be expressed in different DC types in primates and in rodents. In human blood, for example, TLR9 is primarily expressed by the plasmacytoid DC, while, in mice, other subsets of DCs respond to DNA and CPG oligonucleotides. Therefore it remains challenging for DNA vaccines to direct the maturation of human DCs. This maturation can be regarded as a universal platform for vaccine efficacy.

The boosting of DNA-vaccinated individuals with viral vectors may also exploit DCs - again through several mechanisms. The vectors may directly infect DCs or be presented by the exogenous pathway. Some viral vectors may kill infected DCs, leading to uptake and maturation by other DCs as discussed above. In spite of these possibilities, there are few studies of DC function in the setting of DNA prime-viral vector boost vaccination in animals and, as a result, little direct information on whether DCs could be exploited to a greater extent.

Ex vivo-derived $D C$ in cancer vaccines. A new field has emerged in the setting of cancer immunotherapy (see Yu and Restifo, this Perspective series, ref. 13). Human DCs are generated ex vivo from progenitors, charged with antigens from the tumor, and then reinfused to boost a patient's immunity in an antigen-specific manner $(10-12)$. Beyond the goal of developing new therapeutic vaccines that prevent the initial development or recurrence of tumors, the ex vivo DC approach provides an opportunity to investigate many pertinent features of human DCs as natural adjuvants. For example, the way to load DCs with a large spectrum of antigens can be monitored and optimized, the functions of distinct DC subsets can be assessed, and the maturation status of the DCs can be manipulated.

The immunologic impact of DC interactions with specific pathogens. Despite the evident promise of DCs for vaccination, it is important not to overlook the immuneevasive capacities of individual pathogens, many of which can directly disrupt components of DC function. HIV-1 and measles can be cytopathic, particularly following syncytium formation between DCs and T cells. Herpes simplex, cytomegalovirus, lymphocytic choriomeningitis virus, and tumors can block DC functions, including maturation.

The premise of vaccine design is that strong immunity will be able to block the pathogen before it can significantly compromise immunity, even at the level of DCs. Nevertheless, two disquieting aspects of this interaction have come to light, especially in the setting of HIV-1. First, DCs can act like a Trojan horse to transport HIV-1 to T cell sites for replication. First, The DC-restricted lectin DC-SIGN, which is normally used by DCs to bind ICAM- 3 on resting T cells, also binds HIV-1 and enhances its infectivity for T cells following initial uptake into DCs (34). Second, the large amounts of HIV-1 produced during chronic HIV-1 infection are proposed to exploit the normal tolerizing role of immature DCs (33), and to induce T cell deletion and regulatory $\mathrm{T}$ cell formation. HIV-1 virions bind several receptors on the tolerizing, immature form of DCs, including DC-SIGN, CD4, CCR5. In the difficult case of HIV-1, DCs therefore occupy both fronts of vaccine biology, guiding the offense of the pathogen and bolstering the defense of the host. Thus, pathogen interactions with DCs can present a formidable challenge to the development of safe vaccines that target these cells.

\section{Conclusions}

Vaccine design extends beyond the identification of antigens. It needs to harness the immunologic mechanisms that lead to strong and lasting immunity. In many instances, particularly the induction of $\mathrm{T}$ cell-mediated immunity, these mechanisms are controlled by antigen-presenting DCs, potent stimulators of specific $\mathrm{T}$ cell immunity, in tissue culture, in model organisms, and in humans. DCs in essence act as nature's adjuvants to generate immune resistance.

We have outlined three areas of DC biology that might be exploited to improve vaccine efficacy. First, DCs have select receptors for enhancing antigen uptake and processing; these could be targeted to improve the presentation of vaccine antigens to both $\mathrm{CD}^{+} \mathrm{Th} 1$ helper cells and $\mathrm{CD}^{+}$CTLs. Second, DCs undergo a process of terminal differentiation or maturation, typically in response to signaling via TLRs; vaccine-based stimulation of DC maturation is required in addition to antigen capture for DCs to elicit strong T cell immunity. Third, the numbers and migration of DCs in situ can be controlled to improve the selection of particular antigen-responsive lymphocytes. Several newly recognized DC functions pertinent to vaccine design are emerging. DCs or certain DC subsets exert innate functions, such as the production of large amounts of immune-enhancing cytokines. DCs also influence antibody production, control mucosal immunity, and, in the absence of maturation, induce antigen-specific silencing or tolerance. Ironically, because many of the pathogens for which vaccines are desired, especially HIV-1, have the capacity to exploit these cells during their replication or as means to evade immune defenses, DCs contribute to the pathogenesis and protective fronts of vaccine biology.

\section{Acknowledgments}

The authors thank Carol Moberg, John Mascola, and Robert Seder for help with the manuscript. Ralph Steinman is supported by NIH grants AI-13013, AI-40045, AI-40874, and CA-84512, and by Direct Effect. Melissa Pope is supported by NIH grants AI-40877, AI-47681, AI-52060, HD-41757, and HD-41752, and by The Rockefeller Foundation. Melissa Pope is an Elizabeth Glaser Scientist, supported by 
the Elizabeth Glaser Pediatric AIDS Foundation. Our website contains an earlier version of this article with an expanded reference list that extends beyond the background reading below.

1. Letvin, N.L. 2002. Strategies for an HIV vaccine. J. Clin. Invest. In press. 2. Seder, R.A., and Hill, A.V. 2000. Vaccines against intracellular infections requiring cellular immunity. Nature. 406:793-798.

3. Mascola, J.R., and Nabel, G.J. 2001. Vaccines for the prevention of HIV-1 disease. Curr. Opin. Immunol. 13:489-495.

4. Barouch, D.H., Letvin, N.L. 2001. CD8+ cytotoxic T lymphocyte responses to lentiviruses and herpesviruses. Curr. Opin. Immunol. 13:479-482.

5. Berzofsky, J.A., Ehlers, J.D., and Belyakov, I.M. 2001. Strategies for designing and optimizing new generation vaccines. Nat. Rev. Immunol. 1:209-219.

6. Singh, M., and O'Hagan, D. 1999. Advances in vaccine adjuvants. Nat. Biotechnol. 17:1075-1081.

7. Schijns, V.E. 2000. Immunological concepts of vaccine adjuvant activity. Curr. Opin. Immunol. 12:456-463.

8. Pulendran, B., Palucka, K., and Banchereau, J. 2001. Sensing pathogens and tuning immune responses. Science. 293:253-256.

9. Banchereau, J., and Steinman, R.M. 1998. Dendritic cells and the control of immunity. Nature. 392:245-252.

10. Fong, L., and Engleman, E.G. 2000. Dendritic cells in cancer immunotherapy. Annu. Rev. Immunol. 18:245-273.

11. Nestle, F.O., Banchereau, J., and Hart, D. 2001. Dendritic cells: on the move from bench to bedside. Nat. Med. 7:761-765.

12. Steinman, R.M., and Dhodapkar, M. 2001. Active immunization against cancer with dendritic cells: the near future. Int. J. Cancer. 94:459-473.

13. Yu, Z., and Restifo, N.P. 2002. Cancer vaccines: progress reveals new complexities. J. Clin. Invest. In press.

14. Hawiger, D., et al. 2001. Dendritic cells induce peripheral T cell unresponsiveness under steady state conditions in vivo. J. Exp. Med. 194:769-780.

15. Pamer, E., and Cresswell, P. 1998. Mechanisms of MHC class I-restricted antigen processing. Annu. Rev. Immunol. 16:323-358.

16. Matsuda, J.L., and Kronenberg, M. 2001. Presentation of self and microbial lipids by CD1 molecules. Curr. Opin. Immunol. 13:19-25.

17. Mellman, I., and Steinman, R.M. 2001. Dendritic cells: specialized and regulated antigen processing machines. Cell. 106:255-258.

18. den Haan, J.M.M., and Bevan, M. 2001. Antigen presentation to CD8+ T cells: cross-priming in infectious diseases. Curr. Opin. Immunol. 13:437-441.

19. Heath, W.R., Carbone, F.R. 2001. Cross-presentation in viral immunity and self tolerance. Nat. Rev. Immunol. 1:126-134.

20. Sigal, L.J., Crotty, S., Andino, R., and Rock, K.L. 1999. Cytotoxic T-cell immunity to virus-infected non-haematopoietic cells requires presentation of exogenous antigen. Nature. 398:77-80.

21. Somersan, S., and Bhardwaj, N. 2001. Tethering and tickling: a new role for the phosphatidylserine receptor. J. Cell Biol. 155:501-504.

22. Porcelli, S.A., and Modlin, R.L. 1999. The CD1 system: antigen-presenting molecules for $\mathrm{T}$ cell recognition of lipids and glycolipids. Annu. Rev. Immunol. 17:297-329.

23. Gonzalez-Aseguinolaza, G., et al. 2002. NKT cell ligand $\alpha$-galactosylceramide enhances protective immunity induced by malaria vaccines. J. Exp. Med. 195:617-624.

24. Medzhitov, R. 2001. Toll-like receptors and innate immunity. Nat. Rev Immunol. 1:135-145.

25. Akira, S., Takeda, K., and Kaisho, T. 2001. Toll-like receptors: critical proteins linking innate and acquired immunity. Nat. Immunol. 2:675-680.

26. Hemmi, H., et al. 2000. A Toll-like receptor recognizes bacterial DNA. Nature. 408:740-745.

27. Sallusto, F., and Lanzavecchia, A. 1999. Mobilizing dendritic cells for tolerance, priming, and chronic inflammation. J. Exp. Med. 189:611-614.

28. Steinman, R.M., Bona, C., and Inaba, K. 2002. Dendritic cells: important adjuvants during DNA vaccination. In DNA vaccines. $\mathrm{H}$. Ertl, editor. Landes Bioscience. Georgetown, Texas, USA. In press.

29. Lanzavecchia, A., and Sallusto, F. 2001. The instructive role of dendritic cells on T cell responses: lineages, plasticity and kinetics. Curr. Opin. Immunol. 13:291-298.

30. Romani, N., et al. 2001. Migration of dendritic cells into lymphatics. The Langerhans cell example: routes, regulation, and relevance. Int. Rev. Cytol. 207:237-270.

31. Matsuno, K., and Ezaki, T. 2000. Dendritic cell dynamics in the liver and hepatic lymph. Int. Rev. Cytol. 197:83-136.

32. Neutra, M.R., Mantis, N.J., and Kraehenbuhl, J.P. 2001. Collaboration of epithelial cells with organized mucosal lymphoid tissues. Nat. Immunol. 2:1004-1009.

33. Steinman, R.M., and Nussenzweig, M.C. 2002. Avoiding horror autotoxicus: the importance of dendritic cells in peripheral T cell tolerance. Proc. Natl. Acad. Sci. USA. 99:351-358.

34. Kwon, D.S., Gregario, G., Bitton, N., Hendrickson, W.A., and Littman, D.R. 2002. DC-SIGN-mediated internalization of HIV is required for trans-enhancement of T cell infection. Immunity. 16:135-144. 